\title{
NOTES
}

\section{Biochemical Characterization of a Prokaryotic Phenylalanine Ammonia Lyase}

\author{
Longkuan Xiang ${ }^{1}$ and Bradley S. Moore ${ }^{1,2 *}$ \\ College of Pharmacy ${ }^{1}$ and Department of Chemistry, ${ }^{2}$ University of Arizona, Tucson, Arizona 85721
}

Received 6 January 2005/Accepted 3 March 2005

\begin{abstract}
The committed biosynthetic reaction to benzoyl-coenzyme A in the marine bacterium "Streptomyces maritimus" is carried out by the novel prokaryotic phenylalanine ammonia lyase (PAL) EncP, which converts the primary amino acid L-phenylalanine to trans-cinnamic acid. Recombinant EncP is specific for L-phenylalanine and shares many biochemical features with eukaryotic PALs, which are substantially larger proteins by $\sim 200$ amino acid residues.
\end{abstract}

The bacteriostatic agent enterocin is a natural product of the marine bacterium "Streptomyces maritimus" whose biosynthesis involves a number of unusual features $(9,16,17,22)$. Among these is the formation of the rare polyketide synthase starter unit benzoyl-coenzyme A (benzoyl-CoA) (15). The initial biochemical reaction involves the conversion of the amino acid L-phenylalanine to trans-cinnamic acid by the novel bacterial phenylalanine ammonia lyase (PAL; EC 4.3.1.5) EncP (24). Activation of cinnamic acid to its CoA thioester, followed by a single round of $\beta$-oxidation, yields benzoyl-CoA $(7,8,23)$, which primes the enterocin type II polyketide synthase for chain extension with seven molecules of malonyl-CoA (Fig. 1).

Although PAL is a ubiquitous higher-plant enzyme that catalyzes the nonoxidative deamination of phenylalanine to cinnamic acid in the committed step to phenylpropanoid metabolites (6), it has only been encountered in a few bacteria, where it is involved in benzoyl-CoA biosynthesis in " $S$. maritimus" (24) and Sorangium cellulosum (10) and in the biosynthesis of cinnamamide in Streptomyces verticillatus (2). We previously characterized the first prokaryotic PAL-encoding gene (encP) and showed that its inactivation resulted in the abolishment of de novo cinnamic acid and enterocin synthesis in " $S$. maritimus" (12, 24). Enterocin biosynthesis could be restored in encP-inactivated mutants through supplementation with cinnamic or benzoic acid, as well as complementation with plasmid-borne encP. Furthermore, the heterologous expression of the encP gene under the control of the ermE* promoter in Streptomyces coelicolor led to the production of cinnamic acid in the fermented cultures (24). Here we report the biochemical characterization of this novel bacterial PAL, including substrate specificity, $\mathrm{pH}$ dependence, and kinetics, as well as its inhibition with the known plant PAL inhibitor 2-aminoindan2-phosphonic acid (AIP) (1).

Sequence homology, expression, and purification of EncP. The encP gene encodes a 522-amino-acid protein that is considerably smaller than eukaryotic PALs by nearly 200 amino

\footnotetext{
* Corresponding author. Mailing address: College of Pharmacy, University of Arizona, Tucson, AZ 85721-0207. Phone: (520) 6266931. Fax: (520) 626-2466. E-mail: moore@pharmacy.arizona.edu.
}

acid residues. Although sequence homologous to plant PALs such as from Petroselinum crispum (19) (CAA57056; 30\% identical and $48 \%$ similar), it rather shares greater homology to bacterial histidine ammonia lyases (HALs; EC 4.3.1.3) such as from Pseudomonas putida (21) (A35251; 36\% identical and $54 \%$ similar) and to tyrosine ammonia lyase from Rhodobacter capsulatus (13) (Fig. 2). The homology includes the conserved active-site serine residue at position 143 of the phenylalanine/ histidine/tyrosine family of ammonia lyases that is the probable precursor of the modified dehydroalanine residue in the 4methylideneimidazole-5-one prosthetic group $(14,18,21)$. EncP has the greatest sequence homology with AdmH (AAO39102; $63 \%$ identical and $76 \%$ similar), a putative phenylalanine aminomutase involved in andrimid biosynthesis in Pantoea agglomerans that is related to the tyrosine aminomutase $\mathrm{Sgc} 4$ from Streptomyces globisporus $(4,5)$.

The gene encP was PCR amplified from "S. maritimus" cosmid clone pJP15F11 (17) with forward primer 5'-AAAGGAT CCTTCGTCATAGAGCTCGAC-3' (BamHI in bold) and reverse primer 5'-AAAAAGCTTCCAGGTGCTGCTTCAGT
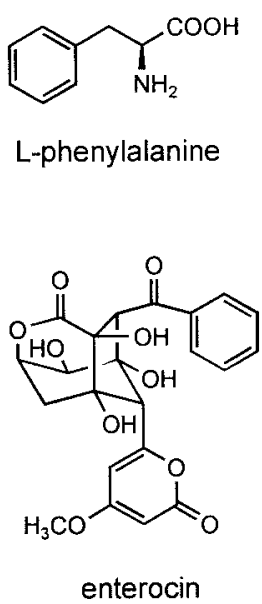

L-phenylalanine

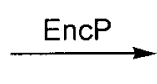

polyketide biosynthesis

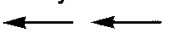<smiles>O=C(O)C=Cc1ccccc1</smiles>

trans-cinnamic acid

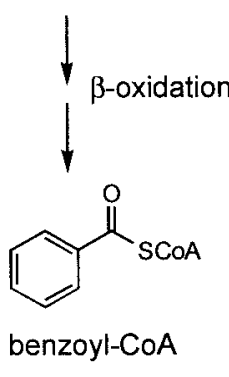

FIG. 1. EncP-catalyzed conversion of phenylalanine to trans-cinnamic acid and biosynthesis of benzoyl-CoA-derived enterocin in "S. maritimus." 


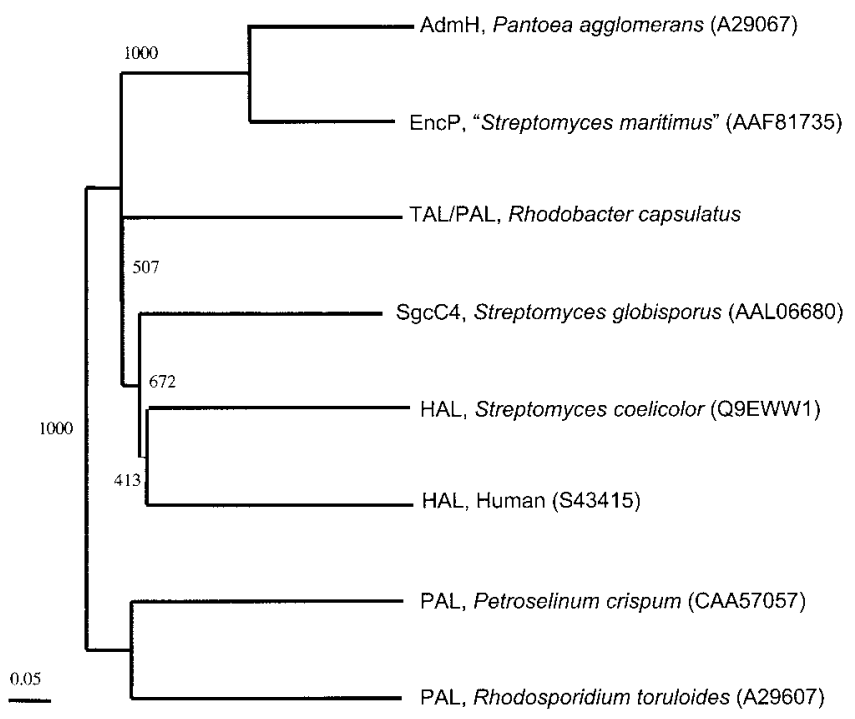

FIG. 2. Relatedness tree of aromatic amino acid ammonia lyases from prokaryotes and eukaryotes. Sequences were retrieved from GenBank (accession numbers are given in parentheses) and aligned with ClustalX (1.83) using the neighbor-joining method.

G-3' (HindIII in bold) and cloned into $\mathrm{pCR}^{\mathrm{R}}$-Blunt (Invitrogen). Its sequence was verified, and it was digested with BamHI and HindIII and cloned into expression plasmid pHIS8 (11). Recombinant EncP was overexpressed as an N-terminal octahistidyl-tagged fusion protein in Escherichia coli BL21 (DE3)/pLysS (Invitrogen). A colony of the plasmid-transformed $E$. coli bacteria was grown overnight in $3 \mathrm{ml} \mathrm{LB}$ broth containing $50 \mu \mathrm{g} / \mathrm{ml}$ kanamycin and $37 \mu \mathrm{g} / \mathrm{ml}$ chloramphenicol at $37^{\circ} \mathrm{C}$. One milliliter of the resultant culture was inoculated into $100 \mathrm{ml}$ TB broth with the same antibiotics in a 500-ml Erlenmeyer flask and grown until the optical density at $600 \mathrm{~nm}$ reached 0.7. After induction with $0.2 \mathrm{mM}$ isopropyl- $\beta$-D-thiogalactopyranoside, the cells were cultured for another $20 \mathrm{~h}$ at $28^{\circ} \mathrm{C}$. The recombinant EncP protein was purified by $\mathrm{Ni}^{2+}$ affinity chromatography over a nickel-nitrilotriacetic acid column. Its mobility upon sodium dodecyl sulfate-polyacrylamide gel electrophoresis corresponded to a mass of $\sim 60 \mathrm{kDa}$, in
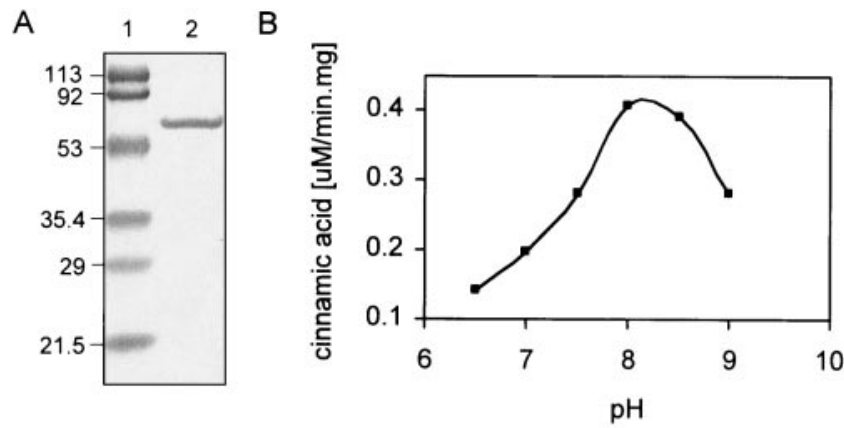

FIG. 3. (A) Sodium dodecyl sulfate-polyacrylamide gel electrophoresis of purified, octahistidyl-tagged EncP. Lane 1, molecular size markers (kDa); lane 2, $\mathrm{His}_{8}$-EncP (calculated molecular mass is 58.7 $\mathrm{kDa}$ ). (B) $\mathrm{pH}$ dependence on the rate of trans-cinnamic acid formation from L-phenylalanine by EncP.
TABLE 1. Kinetic constants of wild-type PALs and EncP mutants ${ }^{a}$

\begin{tabular}{lccc}
\hline \multicolumn{1}{c}{ Enzyme } & $K_{\mathrm{m}}(\mathrm{mM})$ & $k_{\text {cat }}\left(\mathrm{s}^{-1}\right)$ & $\begin{array}{c}k_{\text {cat }} / K_{\mathrm{m}} \\
\left(\mathrm{mM}^{-1} \cdot \mathrm{s}^{-1}\right)\end{array}$ \\
\hline EncP & $0.023 \pm 0.005$ & $4.79 \pm 0.28$ & 208 \\
V83A EncP & $0.120 \pm 0.018$ & $99.58 \pm 1.78$ & 829 \\
V83H EncP & $-b$ & - & - \\
$P$. crispum PAL & $0.12 \pm 0.004$ & $13.5 \pm 0.1$ & 112.5 \\
R. capsulatus TAL/PAL & 1.277 & 15.1 & 11.8 \\
\hline
\end{tabular}

${ }^{a}$ PAL activity was measured by monitoring the formation of trans-cinnamic acid at $280 \mathrm{~nm}$. The kinetic values for EncP are averaged from two independent measurements. The values for $P$. crispum PAL are from reference 19 and the values for $R$. capsulatus TAL/PAL relative to L-phenylalanine are from reference 13.

${ }^{b}$ The activity of $\mathrm{V} 83 \mathrm{H}$ was too low to calculate $K_{m}$ and $k_{\text {cat }}$.

close agreement with the value of 58.7 calculated for the recombinant protein (Fig. 3).

Characterization of recombinant EncP. To confirm that the purified ammonia lyase was indeed specific for L-phenylalanine, the recombinant protein was incubated with phenylalanine, histidine, and tyrosine and monitored spectrophotometrically at $30^{\circ} \mathrm{C}$ by measuring the increase in absorbance at 280 $\mathrm{nm}$ accompanying the formation of the conjugated aryl acid. Imidazole was removed from the elution buffer due to significant enzyme inhibition. The standard assay conditions contained $100 \mathrm{mM}$ Tris- $\mathrm{HCl}$ buffer ( $\mathrm{pH} 8.0$ ), $0.2 \mathrm{mM}$ substrate, and $200 \mu \mathrm{g} / \mathrm{ml} \mathrm{EncP} \mathrm{in} \mathrm{a} \mathrm{1-ml} \mathrm{total} \mathrm{volume.} \mathrm{Incubation} \mathrm{with}$ the substrate L-phenylalanine provided trans-cinnamic acid, which was confirmed by reversed-phase high-performance liquid chromatography (HPLC)-mass spectrometry with an authentic standard. D-Phenylalanine, L-tyrosine, and L-histidine had no detectable activity under similar conditions with up to $2 \mathrm{mM}$ substrate, even when the reaction proceeded overnight and was monitored by HPLC. The pH dependency of the PAL activity was furthermore determined spectrophotometrically and showed a pH optimum of approximately 8.0 (Fig. 3).

Kinetic parameters of cinnamic acid formation. The steadystate kinetic parameters of the recombinant " $S$. maritimus" PAL EncP were determined spectrophotometrically. Two hundred micrograms of the enzyme in $1 \mathrm{ml} 100 \mathrm{mM}$ Tris- $\mathrm{HCl}(\mathrm{pH}$ 8.0) was preincubated at $30^{\circ} \mathrm{C}$ for $5 \mathrm{~min}$ before 0.02 to $0.2 \mathrm{mM}$ L-phenylalanine was added to initiate the 20 -min reaction. $\mathrm{Ki}$ netic constants were calculated from initial velocity measurements at $280 \mathrm{~nm}$ ( $\varepsilon=17,423$ for trans-cinnamic acid $)$ in which product formation was linear over the time periods monitored. The kinetic experiments showed that recombinant EncP had a slightly larger $K_{m}$ and a smaller $k_{\text {cat }}$ than that of PAL from $P$. crispum, as summarized in Table 1 .

The three-dimensional X-ray structures of HAL from $P . p u$ tida $(20,21)$ and, more recently, of PAL from Rhodosporidium toruloides (3) revealed the active-site residues of these tetrameric enzymes that are important for substrate binding, catalysis, and 4-methylideneimidazole-5-one formation. All active-site residues in HAL are present in EncP, except for H83 and $\mathrm{E} 414$, which are replaced with valine and glutamine residues, respectively (24). H83 in HAL is proposed to bind and orient the imidazole moiety of L-histidine at the active site and to stabilize an enzyme-bound cationic intermediate, whereas the carboxylate group of E414 may act as a base in catalysis. To examine the contribution of V83 to cinnamic acid formation 


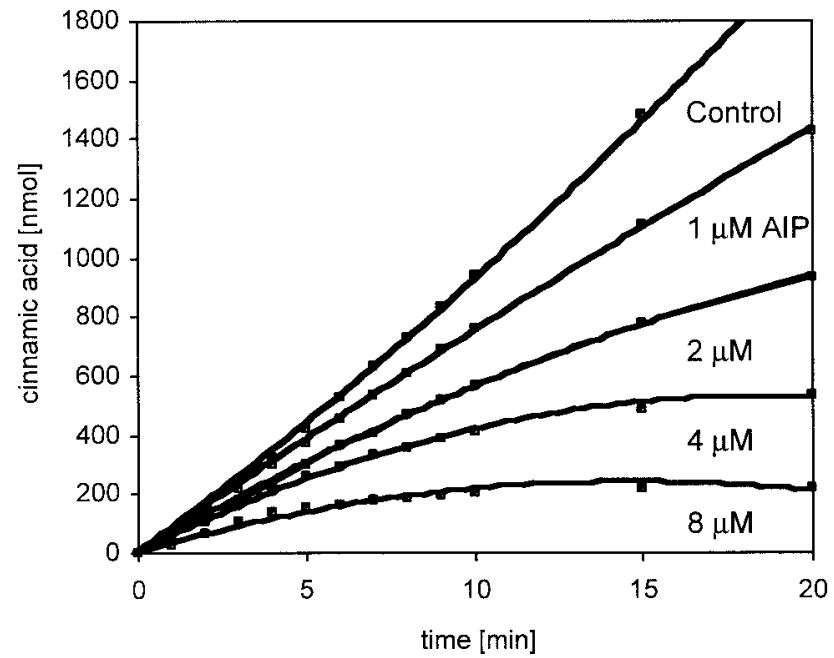

FIG. 4. Assay progress curves in the presence of AIP. EncP was incubated at $40^{\circ} \mathrm{C}$ in $100 \mathrm{mM}$ Tris- $\mathrm{HCl}(\mathrm{pH} 8.0)$ containing $0.4 \mathrm{mM}$ L-phenylalanine and increasing concentrations of AIP. The reaction was started by the addition of the enzyme. Points represent averaged values from duplicate experiments.

by EncP, we generated the V83A and V83H mutants by sitedirected mutagenesis using the QuickChange Multi-Site Directed Mutagenesis method (Stratagene). The V83H mutation was introduced into pHIS8-EncP with primers M13F 5'-CGC CAGGGTTTTCCCAGTCACGACGTTGTAAAACGACGG CCAG-3' and 5'-CCAGGAGAACCTGATCAACGCGCAC GCCACCAACGTGGGGGCG-3' (the underlined bases CA were mutated from GT). The V83A mutation was similarly introduced with primers M13F and 5'-CCAGGAGAACCTG ATCAACGCGGCCGCCACCAACGTGGGGGC-3' (the underlined base $\mathrm{C}$ was mutated from $\mathrm{T}$ ). The mutations were confirmed by DNA sequencing. The mutated encP genes were digested by BamHI-HindIII and cloned separately into pHIS8. In both cases, similar expression levels of the recombinant mutant enzymes showing the same monomeric size as observed with wild-type EncP were measured.

While the V83H mutant lost its PAL activity, the V83A mutant was more active than wild-type EncP (Table 1). The V83A mutant showed a slightly lower affinity to L-phenylalanine with a $K_{m}$ of $120 \mu \mathrm{M}$ versus $23 \mu \mathrm{M}$ for the wild-type enzyme. On the other hand, $k_{\text {cat }}$ was 20 -fold higher, resulting in a mutant that was about six times more active than the wildtype enzyme. The V83H mutant was further tested for HAL activity as the conserved histidine residue in HALs coordinates its imidazole group through a hydrogen bond with that of the bound histidine substrate $(20,21)$. PALs, on the other hand, carry aliphatic residues such as valine and isoleucine at this position, which is consistent with that of EncP, to provide a hydrophobic environment for the benzene ring of its substrate (19). We unfortunately did not observe any HAL activity in the V83H mutant by HPLC analysis, as similarly reported by Rétey and coworkers for the parsley PAL (19).

Kinetic analysis of the inhibition of EncP by AIP. The conformationally restricted phenylalanine analogue AIP has been shown to be an effective inhibitor of plant PAL enzymes both in vivo (25) and in vitro (1). AIP is a competitive inhibitor of
$P$. crispum PAL with a $K_{i}$ of $25 \pm 4 \mathrm{nM}$ and inhibits the enzyme in a time-dependent manner (1). We similarly analyzed the in vitro interaction of AIP with EncP and likewise measured its concentration-dependent inhibition (Fig. 4). The $K_{i}$ of EncP was calculated from the equation $K_{m}{ }^{\prime}=\left(1+[\mathrm{I}] / K_{i}\right)$, where [I] is the concentration of AIP. A $K_{i}$ of $1.91 \pm 0.07 \mu \mathrm{M}$ was obtained for EncP, which was about 76 times higher than that of $P$. crispum PAL.

This work was supported by the NIH (AI47818).

We thank Joseph P. Noel (Salk Institute for Biological Studies, La Jolla, CA) for the vector pHIS8, Jerzy Zon (Wroclaw University, Wroclaw, Poland) for generously providing the inhibitor AIP, and Yoshimitsu Hamano (University of Arizona) for helpful discussions.

\section{REFERENCES}

1. Appert, C., J. Zon, and N. Amrhein. 2003. Kinetic analysis of the inhibition of phenylalanine ammonia-lyase by 2-aminoindan-2-phosphonic acid and other phenylalanine analogues. Phytochemistry 62:415-422.

2. Bezanson, G. S., D. Desaty, A. V. Emes, and L. C. Vining. 1970. Biosynthesis of cinnamamide and detection of phenylalanine ammonia-lyase in Streptomyces verticillatus. Can. J. Microbiol. 16:147-151.

3. Calabrese, J. C., D. B. Jordan, A. Boodhoo, S. Sariaslani, and T. Vannelli. 2004. Crystal structure of phenylalanine ammonia lyase: multiple helix dipoles implicated in catalysis. Biochemistry 43:11403-11416.

4. Christenson, S. D., W. Liu, M. D. Toney, and B. Shen. 2003. A novel 4-methylideneimidazole-5-one-containing tyrosine aminomutase in enediyne antitumor antibiotic C-1027 biosynthesis. J. Am. Chem. Soc. 125:6062-6063.

5. Christenson, S. D., W. Wu, M. A. Spies, B. Shen, and M. D. Toney. 2003. Kinetic analysis of the 4-methylideneimidazole-5-one-containing tyrosine aminomutase in enediyne antitumor antibiotic C-1027 biosynthesis. Biochemistry 42:12708-12718.

6. Hahlbrock, K., and D. Scheel. 1989. Physiology and molecular biology of phenylpropanoid metabolism. Annu. Rev. Plant Physiol. Plant Mol. Biol. 40:347-369.

7. Hertweck, C., A. P. Jarvis, L. Xiang, B. S. Moore, and N. J. Oldham. 2001. A mechanism of benzoic acid biosynthesis in plants and bacteria that mirrors fatty acid $\beta$-oxidation. ChemBioChem 2:784-786.

8. Hertweck, C., and B. S. Moore. 2000. A plant-like biosynthesis of benzoyl-CoA in the marine bacterium "Streptomyces maritimus." Tetrahedron 56:9115-9120.

9. Hertweck, C., L. Xiang, J. A. Kalaitzis, Q. Cheng, M. Palzer, and B. S. Moore. 2004. Context-dependent behavior of the enterocin iterative polyketide synthase: a new model for ketoreduction. Chem. Biol. 11:461-468.

10. Hill, A. M., B. L. Thompson, J. P. Harris, and R. Segret. 2003. Investigation of the early stages in soraphen A biosynthesis. Chem. Commun. 1358-1359.

11. Jez, J. M., J.-L. Ferrer, M. E. Bowman, R. A. Dixon, and J. P. Noel. 2000. Dissection of malonyl-coenzyme A decarboxylation from polyketide formation in the reaction mechanism of a plant polyketide synthase. Biochemistry 39:890-902.

12. Kalaitzis, J. A., M. Izumikawa, L. Xiang, C. Hertweck, and B. S. Moore. 2003. Mutasynthesis of enterocin and wailupemycin analogues. J. Am. Chem. Soc. 125:9290-9291.

13. Kyndt, J. A., T. E. Meyer, M. A. Cusanovish, and J. J. Van Beeumen. 2002. Characterization of a bacterial tyrosine ammonia lyase, a biosynthetic enzyme for the photoactive yellow protein. FEBS Lett. 512:240-244.

14. Langer, B., M. Langer, and J. Rétey. 2001. Methylidene-imidazolone (MIO) from histidine and phenylalanine ammonia-lyase, p. 175-214. In J. P. Klinman and J. E. Dove (ed.), Advances in protein chemistry, vol. 58. Academic Press, Inc., New York, N.Y.

15. Moore, B. S., and C. Hertweck. 2002. Biosynthesis and attachment of novel bacterial polyketide synthase starter units. Nat. Prod. Rep. 19:70-99.

16. Piel, J., C. Hertweck, P. R. Shipley, D. M. Hunt, M. S. Newman, and B. S. Moore. 2000. Cloning, sequencing and analysis of the enterocin biosynthesis gene cluster from the marine isolate "Streptomyces maritimus": evidence for the derailment of an aromatic polyketide synthase. Chem. Biol. 7:943-955.

17. Piel, J., K. Hoang, and B. S. Moore. 2000. Metabolic diversity encoded by the enterocin biosynthesis gene cluster. J. Am. Chem. Soc. 122:5415-5416.

18. Poppe, L. 2001. Methylidene-imidazolone: a novel electrophile for substrate activation. Curr. Opin. Chem. Biol. 5:512-524.

19. Röther, D., L. Poppe, G. Morlock, S. Viergutz, and J. Rétey. 2002. An active site homology model of phenylalanine ammonia-lyase from Pretroselinum crispum. Eur. J. Biochem. 269:3065-3075.

20. Röther, D., L. Poppe, S. Viergutz, B. Langer, and J. Rétey. 2001. Characterization of the active site of histidine ammonia-lyase from Pseudomonas putida. Eur. J. Biochem. 268:6011-6019.

21. Schwede, T. F., J. Rétey, and G. E. Schulz. 1999. Crystal structure of histidine ammonia-lyase revealing a novel polypeptide modification as the catalytic electrophile. Biochemistry 27:5355-5361.

22. Xiang, L., J. A. Kalaitzis, and B. S. Moore. 2004. EncM, a versatile enterocin 
biosynthetic enzyme involved in Favorskii oxidative rearrangement, aldol condensation, and heterocycle-forming reactions. Proc. Natl. Acad. Sci. USA 101:15609-15614.

23. Xiang, L., and B. S. Moore. 2003. Characterization of the benzoyl coenzyme A biosynthesis genes in the enterocin-producing bacterium "Streptomyces maritimus." J. Bacteriol. 185:399-404.
24. Xiang, L., and B. S. Moore. 2002. Inactivation, complementation and heterologous expression of enc $P$, a novel bacterial phenylalanine ammonia-lyase gene. J. Biol. Chem. 277:32505-32509.

25. Zon, J., and N. Amrhein. 1992. Inhibitors of phenylalanine ammonia-lyase: 2-aminoindan-2-phosphonic acid and related compounds. Liebigs Ann. Chem. $625-628$. 